\title{
Multi-center randomized double-blind controlled clinical study of chemotherapy combined with or without traditional Chinese medicine on quality of life of postoperative non-small cell lung cancer patients
}

Ling Xu, HeGen Li, ZhenYe Xu, ZhongQi Wang, LingShuang Liu, JianHui Tian, JianLi Sun, Lei Zhou, YiLin Yao, LiJing Jiao, Wan Su, HuiRu Guo, PeiQi Chen and JiaXiang Liu*

\begin{abstract}
Background: Traditional Chinese medicine (TCM) is a widely applied complementary therapy for cancer patients. It can reduce the chemical drugs induced toxic effects to improve the quality of life (QOL). This study applies the highest quality of clinical trial methodology to examine the role of TCM in improving QOL of postoperative non-small-cell lung cancer patients.

Methods and design: This study is a multi-center, randomized, placebo-controlled, double-blind trial. Four hundred eighty patients will be recruited into seven different research centers in China. These patients that meet the inclusion criteria will be randomized into either a treatment group or a placebo group. Each group will receive treatments of 3weekly chemotherapy with TCM or placebo for four cycles. The primary outcome will involve the evaluation of QOL and the secondary outcome assessments will include two-year disease-free survival rate and disease-free survival. Other efficacy assessments are changes of TCM symptoms and toxicity. Side effects and safety profile of the therapy would be evaluated at the same time. The investigators expect that TCM therapy combined with chemotherapy is superior to chemotherapy solely in terms of QOL improvement and disease-free survival extension. "Intention-to-treat" analysis will include all randomized participants.
\end{abstract}

Discussion: The results from the clinical trial will provide evidence for the effectiveness of chemotherapy combined with or without TCM in QOL of postoperative NSCLC patients.

Trial registration: Clinical Trials.gov (Identifier: NCT01441752).

\section{Background}

Lung cancer is one of the most common malignant tumors in the world, accounting for $26-29 \%$ of all cancer deaths [1]. Increasingly, lung cancer is becoming a global health problem, with greater than 1 million deaths annually attributed to lung cancer [2]. Nonsmall cell lung cancer (NSCLC) accounts for approximately $80 \%$ of all lung cancer diagnoses and more than $75 \%$ of the patients are diagnosed at an advanced stage.

\footnotetext{
*Correspondence: liujiaxiang2012@126.com

Department of Oncology, Longhua Hospital, Shanghai University of TCM, 725 Wanping road, Shanghai 200032, China
}

Consequently, the prognosis for NSCLC continues to remain poor, with a 5 -year survival of $15 \%$ [3].

At present, the high rate of recurrence and metastasis of postoperative non-small cell lung cancer (NSCLC) patients is one of the leading causes resulting in failure of treating lung cancer. More than $35 \%$ of postoperative lung cancer patients with stage I died in 5 years due to recurrence or metastasis; the 5-year survival rate of stage II, IIIa, IIIb was $31 \%, 17.9 \%$ and $11.7 \%$ respectively. The survival rate was improved by $5 \%$ with adjuvant chemotherapy after resection, so regimen consist of platinumbased two chemical medicines are commended as the 
adjuvant chemotherapy for treating postoperative NSCLC patients, but the toxicity and side effects of chemotherapy such as nausea and vomiting, hair loss, and bone-marrow depression can decrease quality of life (QOL) of patients [4-6]. These adverse reactions need to be treated, otherwise which will impact the completion of treatment. Literature and preliminary studies have shown that traditional Chinese medicine (TCM) can prolong survival and improve QOL, but high-level evidence is desperately needed to support this finding [7].

Over the past 10 years, many oncologists focused on survival advantage of adjuvant chemotherapy of postoperative non-small cell lung cancer patients. Data from JBR.10 study showed that overall QOL and functional domain scores are somewhat compromised after thoracotomy and pneumonectomy; but scores improve fairly early in patients who are not receiving chemotherapy [8]. As expected, adjuvant chemotherapy has an immediate negative impact on a number of aspects of QOL in individuals with NSCLC who have undergone resection with curative intent [9]. Chief among these is the symptoms that reflect most of the serious adverse effects associated with platinum-based chemotherapy: fatigue, loss of appetite, nausea, and vomiting. Therefore, QOL in clinical assessment has become more and more prominent in clinical research [10].

Over the past decade, Chinese medicine has made progress in the treatment of lung cancer patients. Chinese medicine has some unique advantages over chemical drugs, such as improving the quality of life, reducing recurrence rate and prolonging overall survival, etc.
Regarding KPS score, traditional Chinese medicine can stabilize and improve the KPS scores after surgery in $82.0 \%$ to $90 \%$ patients $[11,12]$. In addition, it can improve QOL significantly by using NCI-L evaluation. $85 \%$ to $87.5 \%$ of postoperative patients can stabilize and improve the body weight after receiving TCM therapy [11-15]. Two comparative studies from Japan indicated Chinese herbal medicine to be effective in reduction of chemotherapy-induced muscle pain and diarrhea [16,17].

Professor Liu Jiaxiang in Longhua hospital affiliated to Shanghai university of TCM has been doing clinical research on advanced lung cancer patients since the 1960 s. His team have completed four prospective randomized clinical studies supported by China's State Science and Technology. The result showed that disease stable rate was $67.83 \%, 1,3$ and 5-year survival rate was $60.94 \%, 31.86 \%$ and $24.22 \%$ respectively in the treatment group. Median survival was 417 days (13.9 months). The immune function and QOL were improved after treated with combined Chinese and Western medicine compared to the control group [18-25]. This reflected characteristics and therapeutic effect of Chinese medicine on treatment of lung cancer based on syndrome differentiation. But these studies have methodological issues and can't be widely accepted worldwide.

This paper has been compiled to outline the methodology for a randomized-controlled clinical study the effect of chemotherapy combined with or without TCM in QOL of postoperative NSCLC patients that adhere to the CONSORT [26] checklist in its design.

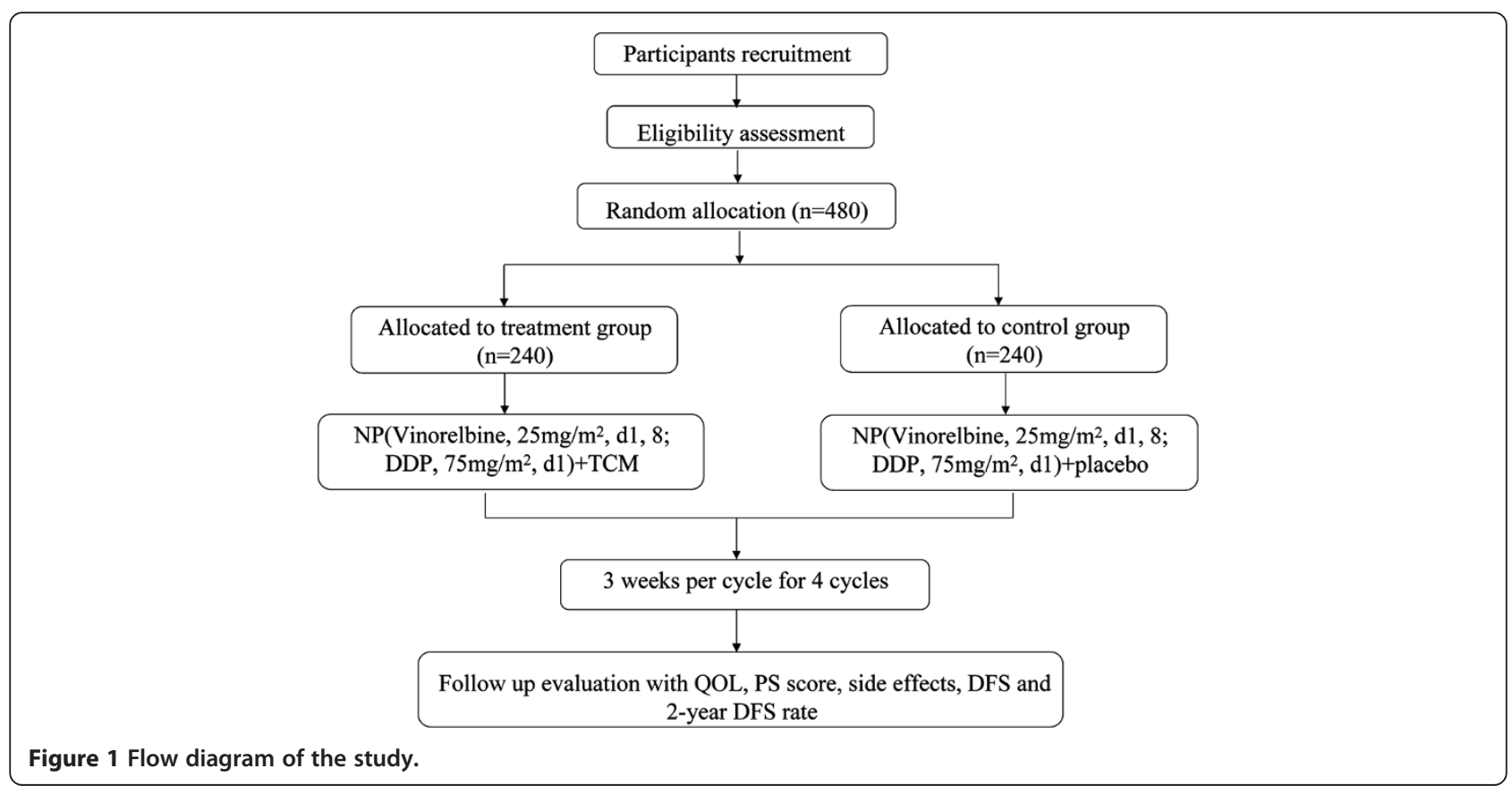




\section{Study aims}

The primary aim of this study is to evaluate the effectiveness of chemotherapy combined with or without TCM in QOL of postoperative NSCLC patients. The secondary aims are to compare the disease-free survival time (DFS), two-year disease-free survival rate and safety of intervention in the two groups.

\section{Methods}

\section{Study design}

This study is a multi-centered, randomized, placebocontrolled, double-blind trial. Subjects from seven clinical research centers in China will engage into the trial. Longhua hospital affiliated to Shanghai university of TCM, Shanghai Chest Hospital affiliated to Shanghai Jiao Tong University, Ruijin Hospital affiliated to Shanghai Jiao Tong University, Huadong hospital affiliated to Fudan university, NO.6 people's hospital affiliated to Shanghai Jiaotong university and Changzheng Hospital of the Second Military Medical University. Eligible participants will be randomly allocated into either of the two groups (chemotherapy with TCM or chemotherapy with placebo) and will receive treatment for 4 cycles (Figure 1).

\section{Randomization}

Dynamic stratified and randomized method is applied in this study. Random numbers will be automatically generated by computer referring to pre-configured stratified factors (stages, pathological types, ages and TCM syndromes). Shanghai Clinical Research Center will provide the random number to each participating center. The block size and treatment-assignment table will not be available to the researchers until the end of the study.

\section{Target population and selection}

NSCLC patients received complete resection with confirmed pathological diagnosis of stage Ib-IIIa; TCM syndromes are Yin deficiency, Qi deficiency, Qi and Yin deficiency, Spleen and Kidney deficiency.

\section{Diagnostic criteria}

Diagnostic criteria for primary bronchial lung cancer: According to the "New compiled diagnosis and treatment norms of common malignant tumors" (Volume IX - primary bronchial lung cancer, the Chinese AntiCancer Association compiled, Beijing: Chinese Union Medical University Press, 1997: 737-781).

TNM stage of primary bronchial lung cancer: According to the staging system of 2009 International Union Against Cancer (UICC), version 7.

\section{Syndrome differentiation criteria}

Syndrome differentiation criteria based on "The Guiding Principles of Clinical Research of New Chinese Medicine (trial)" (China Pharmaceutical Technology Publishing House, 2002) and "Shanghai diagnosis and treatment routine of TCM Syndrome"(Shanghai Municipal Health Bureau edit). Four syndromes of TCM are set as follows:

Qi deficiency syndrome is composed of the following symptoms: coughing, phlegm, poor appetite, spiritlessness and weakness, light and plump tongue; secondary symptoms of spontaneous sweating, loose stool or soft slippery pulse.

Yin deficiency syndrome contains main symptoms of coughing, less phlegm, dry mouth, red tongue; secondary symptoms of the night sweating, heartburn and insomnia, low fever, thread and rapid pulse.

Qi and Yin deficiency syndrome contains main symptoms of coughing, less phlegm, shortness of breath, spiritlessness and weakness, thirst without the desire to drink; secondary symptoms of spontaneous sweating, night sweating, reddish tongue or tongue with teeth marks, and thread and weak pulse.

Spleen and Kidney deficiency syndrome contains main symptoms of coughing, shortness of breath, dyspnea after movements, weakly coughing; secondary symptoms of soreness of loins, tinnitus, loose stool, spiritlessness and weakness, light tongue or tongue with teeth marks, and deep thready and weak pulse.

The diagnosis can be given with the conditions of at least two of the main symptoms and one of the secondary symptoms.

\section{Inclusion criteria}

Meet the diagnostic criteria of primary bronchial lung cancer, and pathologically or cytologically confirmed of squamouscarcinoma, adenocarcinoma, adenosquamous carcinoma or large cell carcinoma of the lung, age between 18 and 75 years old; TCM syndromes are Yin deficiency, Qi deficiency, Qi and Yin deficiency or Spleen and Kidney deficiency, Eastern Cooperative Oncology Group (ECOG) performance status of two or less, Stage Ib $\sim$ IIIa with complete resection, first chemotherapy treatment within 6 weeks after operation, normal hematological function with total neutrophil count $>1.5 \times 10^{9} / 1$ and platelets $>80 \times 10^{9} / \mathrm{l}$, normal liver function and kidney function, voluntarily involved to clinical study and sign informed consent.

\section{Exclusion criteria}

Suffering from other primary malignant tumors; incomplete resection or uncertain to take resection; serious problem of heart, liver or kidney with severe dysfunction; being pregnant or breast-feeding, mental or cognitive disorders that would influence judgment of QOL in 
this study; during the period of adjuvant chemotherapy, or prior chemotherapy; being participating other drug trials; allergy to the drug in the study. Any patient with problems mentioned above will be excluded from this clinical study.

\section{Sample size}

Refer to the results of JRB.10 study, for early lung cancer patients after three-month adjuvant chemotherapy, the QOL score assessed by QLQ-C30 scale decreased 27\% compared with baseline. The sample size of this study is based on the validity of assumptions and clinical experience of the past. Compared to the baseline, it is estimated that the QOL score assessed by QLQ-C30 scale for patients treated with three-month postoperative adjuvant chemotherapy, there was $15 \%$ of patients with no deterioration of QOL combined with TCM than treatment without TCM. Inspection level $\alpha=0.05$, grasp 1 beta $=0.90$, compared with the baseline, the QOL score assessed by QLQ-C30 scale after three-months in the treatment group decline $12 \%$ while $27 \%$ in the control group, made 198 cases of sample size in each group and consider $20 \%$ off rate, it is expected that the research team observed 480 cases $(n=240$ cases) of patients in 2 years to compare QOL of postoperative NSCLC patients by chemotherapy with or without TCM.

\section{Intervention}

Researchers will undertake a one-day training course for this trial. This course will cover the study protocol, the recording method for the clinical record form (CRF), and basic information about clinical research, a researcher's responsibility and research monitoring.

\section{Chemotherapy}

The chemotherapy for NSCLC patients is a combination of Vinorelbine, $25 \mathrm{mg} / \mathrm{m}^{2}$, d1, 8 and DDP, $75 \mathrm{mg} / \mathrm{m}^{2}$, d1 (NP) giving three-weekly for four cycles. For patients who receive fewer than the intended number of cycles of chemotherapy, the study duration will be calculated by the basis of the projected interval.

\section{Chinese herbal medicine}

Prescriptions formulated into granules origin from Professor Liu Jiaxiang in Longhua hospital. Package of granules is made into three types with functions such as benefiting Qi recipe, benefiting Yin recipe and detoxication and resolving masses recipe (Table 1). Each package contained $20 \mathrm{~g}$ of water-soluble herbal granules that were manufactured at a Good Manufacture Practice standard facility (Tian Jiang Ltd, Jiangyin, China). Each package was labeled with a serial number. The prescription form comprised the stock list with both the name and serial number.

\section{Placebo}

It is difficult to define a pure placebo in Chinese herbal medicine where all natural substances are potentially therapeutic. So we compromise the raw materials for the placebo including $10 \%$ of Chinese medicine, food color and artificial flavors. The placebo and therapeutic packages were stored in different cabinets, and only the dispensing technician knew the contents of the packages.

\section{Primary outcome measurement Quality of life}

Quality of life scale: QLQ-LC43 scales made by EORTC will be applied to assess the quality of life of patients. Scoring method is used to assess the results according to the score changes [27]. Scores of general QOL and each field is calculated respectively by international uniform scoring system prior and after intervention. Performance status of patients physical conditions are assessed according to ECOG PS standard before and after each treatment.

\section{Secondary outcome assessments}

Two-year disease-free survival rate: the patients without recurrence and metastasis within 2 years after resection account for percentage of all patients.

Disease-free survival (DFS) :it refers to the interval time either from the first date of medication to that of recurrence and metastasis observed, or death for any reason (record according to the first event). The date of last evaluation will be recorded if patients either have no recurrence and metastasis when reach censored data, or loss of follow-up.

\section{Other outcome assessments}

\section{Evaluation of TCM symptoms changes}

The symptoms scores are made, recorded and calculated based on graded scale of lung cancer symptoms required in "The Guiding Principles of Clinical Research of New Chinese Medicine treating Primary Bronchial Lung Cancer" (2002) issued by the State Drug Administration before and after each treatment. The total scores of single symptom are TCM symptoms scores, and symptoms efficacy assessments are based on the changes before and after treatment.

\section{Safety assessment}

Blood routine, liver and kidney function, urination and stool routine and EKG will be measured before and after treatment to assess the toxicity and side effects of each group according to Common Terminology Criteria for Adverse Events V3.0 (CTC AE) issued by National Cancer Institute (NCI) (http: // ctep.info. nih.gov). 
Table 1 Dosage of Chinese medicine according to different syndromes

\begin{tabular}{llll}
\hline Syndrome Differentiation & \multicolumn{2}{l}{ Chinese Medicine (unit:package) } \\
\cline { 2 - 4 } & Benefiting Qi Recipe & Benefiting Yin Recipe & Detoxication and Resolving Masses Recipe \\
\hline Qi Deficiency & 1 & - & 2 \\
Yin Deficiency & - & 2 & 2 \\
Qi and Yin Deficiency & 1 & 1 & 2 \\
Spleen and Kidney Deficiency & 2 & - & 2 \\
\hline
\end{tabular}

AEs is recorded according to Common Terminology Criteria for Adverse Events V3.0 (CTC AE) made by National Cancer Institute (NCI) (http://ctep.info.nih.gov) after treatment each week.

\section{Adverse events (AE)}

Adverse events including toxicity and side effects should be reported according to Common Terminology Criteria for Adverse Events V3.0 (CTC AE) issued by National Cancer Institute (NCI) (http://ctep.info.nih.gov). All unexpected responses possibly or clearly related to the research will be reported. If serious adverse events (SAE) occur, experimental treatments will be stopped immediately and appropriate treatments will be offered. The type and frequency of adverse events will be reported for each group.

\section{Statistical analysis}

All the clinical data will be saved in computers after collection work and database will be set up by Microsoft Access 2007 software. Statistical analysis will be conducted on an intention-to-treat basis with a 95\% confidence interval using the SPSS 16.0 statistical package. For analysis of baseline characteristics, either twosample $\mathrm{t}$ tests or Wilcoxon rank sum tests for continuous data and Chi-squared tests or Fisher's exact tests for categorical data will be conducted after the test for normality. Kaplan-Meier survival analyses should be used for calculating median survival time (MST), Long-Rank is used between two groups. A significance level of $5 \%$ will be used throughout the analysis. Experts of Shanghai Medical Clinical Research Centre will be invited to manage the statistic analysis.

\section{Discussion}

The aim of this study is to prove that whether chemotherapy combined with TCM treatment can improve patients' quality of life and prolong survival. We have presented the design and protocol for a placebocontrolled, double-blinded RCT that will provide evidence for the effectiveness of chemotherapy combined with or without TCM in QOL of postoperative NSCLC patients. The result will provide support for integrative optimization of the treatment of lung cancer patients.
Competing interests

All the authors have no conflicts of interest.

\section{Authors' contribution}

$L X$, JXL, HGL, ZYX and LJJ were responsible for identifying the research question, designing of the study, obtaining ethics approval, the acquisition of funding and overseeing study implementation. All authors were responsible for the manuscript drafting and have read and approved the final version.

\section{Acknowledgements}

This study is supported by the three-year action project for development of traditional Chinese medicine in Shanghai and Science and Technology

Commission of Shanghai Municipality.

Received: 7 April 2012 Accepted: 18 July 2012

Published: 1 August 2012

\section{References}

1. Jemal A, Siegel R, Xu J, Ward E: Cancer statistics, 2010. CA Cancer J Clin 2010, 60:277-300.

2. Parkin DM, Bray F, Ferlay J, Pisani P: Global cancer statistics, 2002. CA Cancer J Clin 2005, 55:74-108.

3. Spiro SG, Silvestri GA: One hundred years of lung cancer. Am J Respir Crit Care Med 2005, 172:523-529.

4. Siegel R, Ward E, Brawley O, Jemal A: Cancer statistics, 2011. CA Cancer J Clin 2011, 61:212-236.

5. Spiro S, Rudd R, Souhami R, Brown J, Fairlamb D, Gower N, Maslove L, Milroy R, Napp V, Parmar M, Peake M, Stephens R, Thorpe H, Waller D, West $P$ : Chemotherapy versus supportive care in advanced non-small cell lung cancer: improved survival without detriment to quality of life. Thorax 2004, 59(10):828-836.

6. Zhen-Ye X, Jin C-J, Zhou C-C, Wang Z-Q, Zhou W-D, Deng H-B, Zhang M, Wan S, Cai X-Y: Treatment of advanced non-small-cell lung cancer with Chinese herbal medicine by stages combined with chemotherapy. J Cancer Res Clin Oncol 2011, 137:1117-1222

7. Mok TSK, Yeo W, Johnson PJ, Hui P, Ho WM, Lam KC, Xu M, Chak K, Chan A, Wong H, Mo F, Zee B: A double-blind placebo-controlled randomized study of Chinese herbal medicine as complementary therapy for reduction of chemotherapy-induced toxicity. Ann Oncol 2007, 18(4): 768-774.

8. Bezjak A, Lee CW, Ding K, Brundage M, Winton T, Graham B, Whitehead M, Johnson DH, Livingston RB, Seymour L, Shepherd FA: Quality-of-life outcomes for adjuvant chemotherapy in early-stage non-small-cell lung cancer: results from a randomized trial, JBR.10. J Clin Onco 2008, 26 (31):5052-5059.

9. Chen S, Flower A, Ritchie A, Liu J, Molassiotis A, Yu H, Lewith G: Oral Chinese herbal medicine (CHM) as an adjuvant treatment during chemotherapy for non-small cell lung cancer: a systematic review. Lung Cancer 2010, 68:137-145.

10. Cheng $K-F$, Leung P-C: Use of Chinese herbal medicine as an adjuvant for cancer treatment: a randomized controlled dose-finding clinical trial on lung cancer patients. J CANCER THER 2011, 2:91-98.

11. Altekruse SF, Kosary CL, Krapcho M, Neyman N, Aminou R, Waldron W, Ruhl J, Howlader N, Tatalovich Z, Cho H, Mariotto A, Eisner MP, Lewis DR, Cronin $\mathrm{K}$, Chen HS, Feuer EJ, Stinchcomb DG, Edwards BK: SEER cancer statistics review, 1975-2007 [DB/OL]. AAAA, . http://seer.cancer.gov/statistics/ mortality.html 
12. Spiro SG, Silvestri GA: One hundred years of lung cancer. Am J Respir Crit Care Med 2005, 172(5):523-529.

13. Uy KL, Darling G, Wei X, Yi Q-L, De Perrot M, Pierre AF, Waddell TK Johnston MR, Bezjak A, Shepherd FA, Keshavjee S: Improved results of induction chemoradiation before surgical intervention for selected patients with stage IIIA-N2 non-small cell lung cancer. J Thorac Cardiovasc Surg 2007, 134(1):188-193.

14. Pezzetta E, Stupp R, Zouhair A, Guillou L, Taffe P, von Briel C, Krueger T, Ris $H B$ : Comparison of neoadjuvant cisplatin-based chemotherapy versus radiochemotherapy followed by resection for stage III (N2) NSCLC. European I Cardiothorac Surg 2005, 27(6):1092-1098.

15. Cui Y, Shu XO, Gao Y, Wen W, Ruan ZX, Jin F, Zheng W: Use of complementary and alternative medicine by Chinese women with breast cancer. Breast Cancer Res Treat 2004, 85:263-270.

16. Yamamoto K, Hoshiai H, Noda K: Effects of Shakuyaku-kanzo-to on muscle pain from combination chemotherapy with paclitaxel and carboplatin. Gynecol Oncol 2001, 81(2):333-334.

17. Mori K, Kondo T, Kamiyama Y, Kano Y, Tominaga K: Preventive effect of Kampo medicine (Hangeshashin-to) against irinotecan-induced diarrhea in advanced non-small-cell lung cancer. Cancer Chemother Pharmacol 2003, 51:403-406.

18. Liu JX: Clinical observation of 200 cases of syndrome differentiation of traditional Chinese medicine in the treatment of lung cancer. Cancer Res Prev Treat 1978, 2:48

19. Liu JX: Analysis of diagnosis and treatment of 310 primary lung cancer patients. Shanghai J Tradit Chin Med 1985, 10:3.

20. Liu JX: Strengthening body resistance method of treatment of 122 patients with advanced non small cell lung cancer: a prospective study. China J Tradit Chin Med Pharm 1987, 1:11.

21. Liu JX: Clinical and experimental research of strengthening body resistance method in the treatment of advanced primary lung adenocarcinoma. Cancer Res Prev Treat 1990, 17(2):129.

22. Xu ZY, Liu JX: Yin-Yang balancing therapy of traditional Chinese medicine for caner: report of 112 cases of advanced cancer patients with more than one year survival. Shanghai J Tradit Chinese Med 1992, 3:10.

23. Liu JX: Clinical study of treatment of advanced primary lung cancer with traditional Chinese medicine. J Tradit Chin Med 1995, 3:155.

24. Liu JX: Clinical study of treatment with Jifukang for primary non-small cell lung cancer patients. Tumor 2001, 21(6):488-491.

25. Liu JX, Shi ZM, Li HG: Study on treatment of Yifei Kangliu decoction for 271 non-small cell lung cancer patients. Bull Med Res 2003, 32(3):23-24.

26. Altman DG, Schulz KF, Moher D, Egger M, Davidoff F, Elbourne D, Gøtzsche $\mathrm{PC}$, Lang T: The revised CONSORT statement for reporting randomized trials: explanation and elaboration. Ann Intern Med 2001, 134:663-694.

27. Wan CH, Zhang CZ, Tu X, Feng CY, Tang W, Luo JH, Meng Q: Validation of the simplified Chinese version of the quality of life instrument EORTC QLQ-LC43 for patients with lung cancer. CANCER INVE 2008, 26(5):504.

doi:10.1186/1472-6882-12-112

Cite this article as: Xu et al:: Multi-center randomized double-blind controlled clinical study of chemotherapy combined with or without traditional Chinese medicine on quality of life of postoperative non-small cell lung cancer patients. BMC Complementany and Alternative Medicine 2012 $12: 112$

\section{Submit your next manuscript to BioMed Central and take full advantage of:}

- Convenient online submission

- Thorough peer review

- No space constraints or color figure charges

- Immediate publication on acceptance

- Inclusion in PubMed, CAS, Scopus and Google Scholar

- Research which is freely available for redistribution 